\title{
Development and Research of Data Acquisition System Cen $\mathrm{Li}^{1}$ \\ ${ }^{1}$ Jishou University, Zhangjiajie College, Hunan, 427000 \\ hunter2011@foxmail.com
}

Keywords: Data Acquisition System; Communications; Data Acquisition

\begin{abstract}
The process of data acquisition system to obtain data information is carried out from a plurality of signals. The system is a high degree of automation equipment, it not only can real-time data acquisition, real-time control and status display, it also can be automatically stored, signal preprocessing functions. The first part describes the development process of the data acquisition system, the second section describes the data acquisition abroad of the current situation and problems, and the third part describes the laboratory, logistics and supply chain, marine station and seismic data acquisition application.
\end{abstract}

\section{Introduction}

Data acquisition system originated in the 1950s, and data acquisition systems for the US military test system, the test does not depend on the relevant test documentation, with unskilled personnel can operate, highly functional testing with a test instrument. Data acquisition system can be a lot of testing tasks. In the late 1960s, the data acquisition system gradually into the Chinese market, which now makes data acquisition system with many special features.

In the 1970s, with the development of the microcomputer, the acquisition and instrumentation combined with each other to form a current database collection system, data acquisition system with good performance, it is more than a traditional automatic detection and data acquisition systems easier to use. From the 1970s, the development of data acquisition systems can be divided into two categories, through the corresponding bus companies and research institutions can make use of the data acquisition system for data collection. The original data acquisition system has been greatly changed, industrial computers and microcontrollers, software management, you can reduce the cost of the system, work load system is also greatly reduced, while the enhanced data processing capability has been greatly improved.

\section{Status of data acquisition}

The Meanings of Data Acquisition. Data acquisition device is a portable analyze, its features include recording, analysis and recording functions. Vibration sensors and process sensors installed in the machinery and equipment, the measurement signal as an input basis, the formation of a detection system in a variety of measurement and analysis techniques and display format, mainly used for diagnosis of mechanical equipment fault diagnosis and regular circuits in. It can form an independent monitoring and diagnostic systems, it is computer-aided diagnostic device equipment in a secondary diagnosis.

The Status Quo And Problems of Data Acquisition. In the 1980s and 1990s, from the late 20th century, a number of equipment manufacturers in our country has developed a variety of data collection, including the single-channel SP201, SC247, dual channel EG3300, YE5938 subminiature 911902 and 921 in order to meet maintenance the initial level of spectral analysis of management and external data acquisition devices, static signal acquisition, including the SMC-9012 and the support package in two parts.

However, there are still national data collection and data acquisition system, which is mainly reflected in some of the gap between technology:

(1) Due to the limited level sensor, the frequency range is not wide analysis, the relative should bring some difficulties, mainly for low speed diagnosis or bearing problems; 
(2) data collection, storage memory is small, so that the function of data acquisition and signal processing is not very strong in this area can only do some simple diagnosis, accurate diagnosis, then must be done on the computer, so a functional site diagnosis is relatively weak.

(3) Software Level equipment maintenance management stage management is still in the spectral analysis and equipment. Mechanical fault diagnosis expert system needs to be improved.

Data acquisition is a key element for the entire plant to realize automation and an important factor in testing accuracy, speed, and realize the function of the cost of data collection. It is also moving in this direction. High-speed real-time data acquisition is even more important in the following areas: explosives detection, medical equipment, such as CT, MRI, rapid manufacturing process. In these industries, the demand for high speed data acquisition is far more than the actual level. The needs of users, and promote the development of technology and the emergence of new products. Therefore, high speed data acquisition still has a larger space for development.

\section{Data Acquisition Applications}

According to different uses of the data acquisition, the data acquisition system can be divided into two types according to use the function: An online data acquisition, the other is a portable data acquisition. Online data acquisition systems can be divided into two types one is the other is a modular desktop. Desktop and portable data collector is an AC power supply; modular data acquisition, which is the most commonly used data acquisition and transmission system, which is based on the DC power supply. Data acquisition applications involve a number of area, the following is a description of a number of areas of application.

The Applications of Data Acquisition in Laboratory. First, the data acquisition applied physics laboratory is "science workshop" Science Workshop is a new system, which is produced by the US PASSCO company. "Scientific Studio" consists of three parts:

(1) Sensors for technical physics experiments of various physical quantities of data acquired by the cash of sensing technology.

(2) The incoming sensor data acquisition signal to the computer software, which is done through the computer interface, its highest sampling rate of up to 25 million times/S.

(3) Application software can support English and Chinese.

Science Workshop data acquisition several applications:

(1) Data collection and computers through binding, not only can improve the accuracy of the test, but also to avoid the errors that appear in traditional tests, and can automatically output the result of the measurement data and testing. (2) by physical experiments instantaneous change can be seen in data acquisition and low visibility.

(3) It can not be directly observed in some physical changes have been made in the control experiment.

(4) By way of trials to test the data acquisition of physical concepts prone to error.

(5) To have a sense of outdoor activities, while the use of physical

Data Acquisition Applications in the Logistics Supply Chain Management. Another type of portable data collector is designed to scan the bar code symbol. When reading, the scanner scans the bar code symbol, and data collection and the display is reversed. It has its own internal memory can store a certain amount of data, the use of battery-powered or DC can use for performing communication and data transfer between the computer and the scanner to the computer. Most portable barcode collection device in order to meet a variety of needs, self-programming capabilities have, of course, its own application is a powerful special equipment. With the development of the logistics industry, a growing number of such enterprises will put portable bar code data collection for practical work, of course, a lot of logistics enterprises in China is also widely portable bar code collection for warehouse management and transportation management. Application data acquisition system can not only help logistics companies to better manage the implementation of network automation, more cost and time savings, reducing the workload of workers to reduce costs, while improving inventory accuracy. 
Data Acquisition Application in Marine Data Acquisition Station. Automatic detection of data acquisition system in general is mainly used in the marine station, through the automatic detection of data acquisition in order to realize the function of the marine station automation. So far, data acquisition automatic detection function in the marine station in this area, and the use of foreign data has commonality (YRON SU8200, YRON SU9000, DT50, DT500), features include modular, simulation, flexible and stable and reliable Wait. In different environments and use, their characteristics are not the same. The following is a combination of ocean station system features automatic detection function data acquisition system features summarized:

(1) strong, weak and electromagnetic interference, lightning protection can be effective; (2) design module easy maintenance and low power consumption; (3) to facilitate the temperature, barometric pressure, wind, humidity, rain, water, salt and other signal access into; (4) has a supplementary charge, print, send and control functions; (5) reliable data acquisition software and cable transmission, wireless, modem, satellite communications, rich peripheral interfaces; (8) AC and DC, under-voltage protection, reliable power supply can also be solar access.

Data Acquisition Application in Seismic Data Acquisition of Earthquake. Critical equipment data Seismic Network is dedicated seismic data acquisition, seismic data acquisition main function is analog voltage output digital. The basic parameters of seismic data acquisition data is a critical foundation for Digital Seismic Network technical parameters.

\section{Acknowledgements}

Project: A Project Supported by Scientific Research Fund of Hunan Provincial Education Department

Project number: 15C1121

\section{References}

[1] P. Ruan, Z. Zhang. DSP and Communication Data Acquisition System PCI Bus. Based on Electronic Technology, 2012, (5): 1-78.

[2] P. Yan. Communication and Industrial Data Acquisition. Modern Manufacturing, 2012 (13): 20-23.

[3] Z.G. Gu. Data Acquisition Developments. China Set Up Various Projects, 2010 (3): 38-39.

[4] Z.B. Peng, Y.j. Du. Commonly Used In Distributed Data Acquisition Performance, Introduce. Aviation Measurement Technology, 2011 (6).

[5] L.F. Lai, X.J. Wang. Data Logger Used To Explore Physics Inquiry Learning. Physics Experiments, 2013 (7): 32-33. 